\title{
HIGHER WHITEHEAD GROUPS OF CERTAIN BUNDLES OVER SEIFERT MANIFOLDS
}

\author{
A. J. NICAS AND C. W. STARK
}

\begin{abstract}
Vanishing results for $\mathrm{Wh}_{i}\left(\pi_{1} M\right) \otimes R(R=\mathbf{Z}$. $\mathbf{Q}$, or $\mathbf{Z}[1 / 2])$ are obtained when $M$ is a closed aspherical manifold which is the total space of a bundle over an insufficiently large Seifert manifold with infinite fundamental group of hyperbolic type. Allowable fibers include Riemannian flat manifolds and closed aspherical manifolds with poly- $\mathbf{Z}$ fundamental groups. Corollaries. concern the homotopy groups of the group TOP $(M)$ of self-homeomorphisms of $M$.
\end{abstract}

Let $R$ be a subring of the rational numbers, $n$ a nonnegative integer, and $N$ a connected manifold.

HyPOTHESIS $\mathrm{A}(n, R) . \mathbf{Z} \pi_{1} N$ is a right regular Noetherian ring and $\mathrm{Wh}_{j}\left(\pi_{1} N\right) \otimes R$ $=0$ for $0 \leqslant j \leqslant n$.

$N$ satisfies Hypothesis $\mathrm{A}(\infty, R)$ if $N$ satisfies Hypothesis $\mathrm{A}(n, R)$ for all $n$. It is known that if $\pi_{1} N$ is a poly-Z group then $N$ satisfies $\mathrm{A}(\infty, \mathbf{Z})$. If $\pi_{1} N$ is a Bieberbach group then $N$ satisfies $\mathrm{A}(1, \mathbf{Z}), \mathrm{A}(3, \mathbf{Z}[1 / 2])$, and $\mathrm{A}(\infty, \mathbf{Q})$ [FH1, N2, N3]. It is conjectured that if $\pi_{1} N$ is a Bieberbach group then $N$ satisfies $\mathrm{A}(\infty, \mathbf{Z})$.

Waldhausen's results on the $K$-theory of generalized free products [W], especially Corollaries 17.1.3 and 17.2.3, yield the following lemma. Recall that the $j$ th Whitehead group of a group $G$ is the $j$ th homotopy group of a space $\mathrm{Wh}^{\mathbf{Z}}(G)$ and that these corollaries establish homotopy Cartesian squares involving these Whitehead spaces.

LemMa. Let $M$ be the total space of a fiber bundle over a compact, connected manifold $K$ with fiber $N$. Assume that $N$ satisfies Hypothesis $\mathrm{A}(n, R)$. If $K$ is a surface other than $S^{2}$ or $\mathbf{R} P^{2}$, of if $K$ is a Haken 3-manifold, then for $0 \leqslant j \leqslant n, W_{h_{j}}\left(\pi_{1} M\right)$ $\otimes R=0$.

Proof. An $N$-bundle over a surface other than $S^{2}$ or $\mathbf{R} P^{2}$ is built up from copies of $N \times D^{2}$ by amalgamating along copies of $N \times D^{1}$ or $N$-bundles over $S^{1}$, while an $N$-bundle over a Haken 3-manifold is built up from copies of $N \times D^{3}$ by amalgamating along $N$-bundles over incompressible surfaces (which are not $S^{2}$ or $\mathbf{R} P^{2}$ ). The arguments for the two cases are essentially the same.

When the base of the bundle is a surface, the integral group rings for the amalgamating subgroups are $\mathbf{Z} \pi_{1} N$ or twisted Laurent extensions of $\mathbf{Z} \pi_{1} N$ : in either

Received by the editors March 8, 1983.

1980 Mathematics Subject Classification. Primary 18F25: Secondary 57T20.

(1)1984 American Mathematical Society $0(0) 2-9939 / 84 \$ 1.0()+\$ .25$ per page 
case the group ring is right regular Noetherian (and hence coherent). This implies, by Corollary 4.2 of [W], that $\mathbf{Z} \pi_{1} M$ is right regular coherent when $K$ is a surface, so Corollaries 17.1.3 and 17.2.3 of [W] are applicable when $K$ is a surface or Haken 3-manifold.

These corollaries give homotopy Cartesian squares with the Whitehead space of a free product with amalgamations or an HNN extension as the lower right-hand corner of the square. Our hypothesis is that, after tensoring with $R$, the other three spaces in the square are $n$-connected. This implies that the lower-hand space in the square is also $n$-connected after tensoring with $R$. (To verify 0 -connectedness, use the argument on p. 250 of [W] based on the Bass-Heller-Swan inclusion $\mathrm{Wh}_{0}(G) \hookrightarrow$ $\mathrm{Wh}_{1}(G \times \mathbf{Z})$.) Repeated applications of this $n$-connectedness observation complete the proof of the Lemma.

Proposition. Let $\pi$ be a group and $f: \pi \rightarrow G$ an epimorphism onto a finite group, $R$ a subring of the rational numbers, and $n$ a nonnegative integer. Suppose that for every hyperelementary subgroup $H$ of $G$ the higher Whitehead groups of $f^{-1}(H)$ satisfy $\mathrm{Wh}_{j}\left(f^{-1}(H)\right) \otimes R=0$ for $0 \leqslant j \leqslant n$. Then $\mathrm{Wh}_{j}(\pi) \otimes R=0$ for $0 \leqslant j \leqslant n$.

PROOF. By $[\mathbf{W}]$ there is a long exact sequence for any group $\Gamma$ :

$(*) \quad \rightarrow \mathrm{Wh}_{j+1}(\Gamma) \rightarrow h_{j}\left(B \Gamma ; K_{\mathbf{Z}}\right) \stackrel{l_{j}}{\rightarrow} K_{j}(\mathbf{Z} \Gamma) \rightarrow \mathrm{Wh}_{j}(\Gamma) \rightarrow \cdots \rightarrow \mathrm{Wh}_{0}(\Gamma) \rightarrow 0$

where $h_{j}\left(; K_{\mathbf{Z}}\right)$ is the generalized homology theory arising from the spectrum $K_{\mathbf{Z}}$ for algebraic $K$-theory and $B \Gamma$ is the classifying space of $\Gamma$. This sequence remains exact when tensored with $R$. From (*) it follows that the conclusion of the theorem is equivalent to the statement; $l_{j}$ is an isomorphism for all $j$ such that $0 \leqslant j \leqslant n-1$ and an epimorphism for $j=n$.

If $H$ is a subgroup of $G$ define

$$
m_{j}(H)=h_{j}\left(B f^{-1}(H) ; K_{\mathbf{Z}}\right) \otimes R, \quad k_{j}(H)=K_{j}\left(\mathbf{Z} f^{-1}(H)\right) \otimes R
$$

and if $H$ and $K$ are subgroups of $G$ and $g \in G$ are such that $g H g^{-1} \subset K$, let ( $H, g, K$ ) be the homomorphism $H \rightarrow K$ given by conjugation by $g$.

Given $I=(H, g, K)$, there is an induction map $I_{*}: k_{j}(H) \rightarrow k_{j}(K)$ ("induced map") and a restriction map $I^{*}: k_{j}(K) \rightarrow k_{j}(H)$ ("transfer"). There is also an induction map $I_{*}: m_{j}(H) \rightarrow m_{j}(K)$ corresponding to the map in homology induced by $B f^{-1}(H) \rightarrow B f^{-1}(K)$ and a restriction map $I^{*}: m_{j}(K) \rightarrow m_{j}(H)$ corresponding to the homology transfer. According to [FH2], $l_{j}: m_{j}(H) \rightarrow k_{j}(H)$ is natural with respect to induction and restriction, and $k_{j}(\mathrm{)})$ is a Frobenius module over Swan's Frobenius functor $G_{0}() \otimes R$, where $G_{0}(H)$ is the Grothendieck group of integral representations of $H$.

Let $C$ be the collection of hyperelementary subgroups of $G$. Define $m_{j}(C)=$ $\bigoplus_{H \in C} m_{j}(H)$ and $k_{j}(C)=\bigoplus_{H \in C} k_{j}(H)$. Consider the following commutative diagrams: 


$$
\begin{array}{crrrrr}
m_{j}(C) & \stackrel{l_{j}}{\rightarrow} & k_{j}(C) & m_{j}(C) & \stackrel{l_{j}}{\rightarrow} & k_{j}(C) \\
I_{*} \downarrow & & \downarrow I_{*} & I^{*} \uparrow & & \uparrow I^{*} \\
m_{j}(G) \stackrel{l_{j}}{\rightarrow} & k_{j}(G) & m_{j}(G) & \stackrel{l_{j}}{\rightarrow} & k_{j}(G) \\
I_{*}=\sum_{H \in C}(H, e, G)_{*} & I^{*}=\prod_{H \in C}(H, e, G)^{*}
\end{array}
$$

Since $G_{0}() \otimes R$ satisfies hyperelementary induction [Sw], it follows from [Dr, Proposition 1.2] (also see [N, Theorem 6.2.7]) that $I_{*}: k_{j}(C) \rightarrow k_{j}(G)$ is surjective and $I^{*}: k_{j}(G) \rightarrow k_{j}(C)$ is injective. By [N, Lemma 6.2.8] $I_{*}: m_{j}(C) \rightarrow m_{j}(G)$ is surjective and $I^{*}: m_{j}(G) \rightarrow m_{j}(C)$ is injective. By hypothesis $l_{j}: m_{j}(C) \rightarrow k_{j}(C)$ is an isomorphism for $0 \leqslant j \leqslant n-1$ and an epimorphism for $j=n$. A diagram chase reveals that $l_{j}: m_{j}(G) \rightarrow k_{j}(G)$ is an isomorphism for $0 \leqslant j \leqslant n-1$ and an epimorphism for $j=n$, completing the proof of the Proposition.

Let $K^{3}$ be one of the insufficiently large Seifert manifolds with three exceptional orbits over $S^{2}$ given by the invariants $\left(b ;(0,0,0,0):\left(\alpha_{1}, \beta_{1}\right),\left(\alpha_{2}, \beta_{2}\right),\left(\alpha_{3}, \beta_{3}\right)\right)$ in the notation of [O]. Let $\pi_{1} K^{3} \rightarrow Q\left(\alpha_{1}, \alpha_{2}, \alpha_{3}\right)$ be the quotient by the image in the fundamental group of any regular fiber: here $Q\left(\alpha_{1}, \alpha_{2}, \alpha_{3}\right)$ is the orientation-preserving subgroup of a triangle group.

MAIN THEOREM. Let $M$ be the total space of a bundle with fiber $N$ and base $K^{3}$, where $K^{3}$ is one of the Seifert manifolds described above. If $\alpha_{1}^{-1}+\alpha_{2}^{-1}+\alpha_{3}^{-1}<1$ and $N$ satisfies Hypothesis $\mathrm{A}(n, R)$, then $\mathrm{Wh}_{j}\left(\pi_{1} M\right) \otimes R=0$ for $0 \leqslant j \leqslant n$.

Proof. This argument is essentially that of $[\mathbf{P}]$ and relies on the fact that $Q=Q\left(\alpha_{1}, \alpha_{2}, \alpha_{3}\right)$ is a hyperbolic triangle group if $\alpha_{1}^{-1}+\alpha_{2}^{-1}+\alpha_{3}^{-1}<1$. In [P and S] it is shown that $Q$ has an epimorphism $h: Q \rightarrow G$ to a nonhyperelementary finite group $G$. Composition gives an epimorphism $f: \pi_{1} M \rightarrow \pi_{1} K \rightarrow Q \stackrel{h}{\rightarrow} G$.

Let $H$ be a subgroup of $G$. If the covering space of $M$ corresponding to $f^{-1}(H)$ is an $N$-bundle over a Haken manifold (i.e. if $h^{-1}(H)$ is not a triangle group), then the Lemma is applicable. As $H$ runs over the hyperelementary subgroups of $G$, though, some of the $h^{-1}(H)$ 's may be triangle subgroups of $Q$, so this observation is not enough to finish the proof. However, hyperbolic triangle groups contain only finitely many triangle subgroups, so we may induce on the number $t(Q)$ of proper triangle subgroups in $Q$. If $t(Q)=0$ then no $h^{-1}(H)$ is a triangle group and the Proposition and Lemma show that $\mathrm{Wh}_{j}\left(\pi_{1} M\right) \otimes R=0$ for $0 \leqslant j \leqslant n$. If $t(Q) \geqslant 1$, then for any $h^{-1}(H)$ which is a triangle group, $t\left(h^{-1}(H)\right)<t(Q)$, so the Proposition and the inductive hypothesis imply $\mathrm{Wh}_{j}\left(\pi_{1} M\right) \otimes R=0$ for $0 \leqslant j \leqslant n$.

Let $M$ and $N$ be as in the Main Theorem. Suppose $N$ satisfies the additional

Hypothesis B. $N$ is a closed aspherical manifold and $S_{\mathrm{TOP}}\left(N \times I^{j}, \partial\right)=0$ for $j+\operatorname{dim}(N) \geqslant 6$, where $S_{\mathrm{TOP}}\left(N \times I^{j}, \partial\right)$ is the structure set of topological surgery [KS].

An interesting class of manifolds which satisfy Hypothesis B is the closed aspherical manifolds with torsion-free poly- (finite or infinite cyclic) fundamental 
group [FH3]. This class includes closed flat Riemannian manifolds and closed manifolds with poly-Z fundamental group. Suppose $N$ satisfies Hypothesis $\mathrm{A}(\infty, Q)$ and B (for example, take $N$ to be a closed Riemannian flat manifold or a closed aspherical manifold with poly- $\mathbf{Z}$ fundamental group). By the Main Theorem, $\mathrm{Wh}_{j}\left(\pi_{1} M\right) \otimes \mathbf{Q}=0$ for all $j$, and by the main theorem of [S], $M$ will also satisfy Hypothesis B in many cases, including these:

(a) $\alpha_{1}, \alpha_{2}$ and $\alpha_{3}$ are all odd, or

(b) an odd prime $p$ divides one of the $\alpha$ 's, say $\alpha_{1}$, and the group $Q\left(\alpha_{1} / p, \alpha_{2}, \alpha_{3}\right)$ is also a hyperbolic group of motions.

Let $\operatorname{TOP}(M)$ be the topological group of self-homeomorphisms of $M$ and let $m=\operatorname{dim}(M)$. Suppose $M$ has the properties established for the examples considered above: $M$ satisfies Hypothesis B and all the Whitehead groups of $M$ vanish when tensored with the rationals. Theorem 4.5(B) of [FH2] now yields a computation of the rational homotopy groups $\pi_{i}(\operatorname{TOP}(M)) \otimes \mathbf{Q}$ for $1 \leqslant i \leqslant \phi_{2}(m)$, where $\phi_{2}(m)$ is the stable range for topological pseudoisotopy.

Corollary 1 . For $1 \leqslant i \leqslant \phi_{2}(m)$,

$$
\pi_{i}(\operatorname{TOP}(M)) \otimes \mathbf{Q}= \begin{cases}\operatorname{center}\left(\pi_{1} M\right) \otimes \mathbf{Q}, & i=1, \\ \bigoplus_{j=1}^{\infty} H_{(i+1)-4 j}(M, \mathbf{Q}), & i>0, \text { m odd }, \\ 0, & i>0, \text { m even } .\end{cases}
$$

REMARK. The theorem of Farrell and Hsiang quoted above, while stated for the differentiable category in [FH2], is equally valid in the topological category. If $M$ is smoothable, Corollary 1 is true for the diffeomorphism group $\operatorname{Diff}(M)$ in place of $\operatorname{TOP}(M)$ provided $1 \leqslant i \leqslant \phi_{1}(m)$, where $\phi_{1}(m)$ is the stable range for smooth pseudoisotopy.

Now suppose $N$ is smoothable and satisfies Hypotheses $\mathrm{A}(1, \mathbf{Z}), \mathbf{A}(3, \mathbf{Z}[1 / 2])$, and B (again this will be the case if $N$ is a closed flat Riemannian manifold or if $N$ is a closed aspherical manifold with $\pi_{1} N$ poly-Z). By the Main Theorem and the main theorem of [S], $M$ satisfies Hypothesis $\mathrm{B}$ and

$$
0=\mathrm{Wh}_{0}\left(\pi_{1} M\right)=\mathrm{Wh}_{1}\left(\pi_{1} M\right)=\mathrm{Wh}_{2}\left(\pi_{1} M\right) \otimes \mathbf{Z}[1 / 2]=\mathrm{Wh}_{3}\left(\pi_{1} M\right) \otimes \mathbf{Z}[1 / 2] .
$$

The following theorem, which is a consequence of the parametrized surgery theory of [HS], was proved in [N3] and applies to $M$ as above:

THEOREM. Suppose $M^{m}, m \geqslant 6$, is a smoothable closed aspherical manifold satisfying Hypothesis $\mathrm{B}$ and

$$
\mathrm{Wh}_{0}\left(\pi_{1} M\right)=\mathrm{Wh}_{1}\left(\pi_{1} M\right)=\mathrm{Wh}_{2}\left(\pi_{1} M\right) \otimes \mathbf{Z}[1 / 2]=\mathrm{Wh}_{3}\left(\pi_{1} M\right) \otimes \mathbf{Z}[1 / 2]=0 \text {. }
$$

\section{Then}

(a) There is a normal abelian subgroup $H \subset \pi_{0}(\mathrm{TOP}(M))$ consisting entirely of 2-torsion such that $\pi_{0}(\operatorname{TOP}(M)) / H \cong \operatorname{Out}\left(\pi_{1} M\right)$, where $\operatorname{Out}\left(\pi_{1} M\right)$ is the group of outer automorphisms of $\pi_{1} M$.

(b) $\pi_{1}(\mathrm{TOP}(M)) \otimes \mathbf{Z}[1 / 2] \cong \operatorname{center}\left(\pi_{1} M\right) \otimes \mathbf{Z}[1 / 2]$. 
Remark. Part (b) depends on the computation by K. Igusa and R. K. Dennis of the kernel of Igusa's map $\chi: \pi_{1}\left(P_{\text {diff }}^{s}(M)\right) \rightarrow \mathrm{Wh}_{3}\left(\pi_{1} M\right)$, where $P_{\text {diff }}^{s}(M)$ is the space of stable smooth pseudoisotopies of $M[\mathbf{D I}]$.

\section{REFERENCES}

[DI] R. K. Dennis and K. Igusa, Hochschild homology and the second obstruction for pseudoisotopy, Proc. $K$-theory Conf. (Oberwolfach, 1980), Lecture Notes in Math., Vol. 967, Springer-Verlag, Berlin and New York, 1982.

[Dr] A. W. M. Dress, Induction and structure theorems for orthogonal representations of finite groups, Ann. of Math. (2) 102 (1975), 291-325.

[FH1] F. T. Farrell and W.-C. Hsiang, The topological Euclidean space form problem, Invent. Math 45 (1978), 181-192.

[FH2] On the rational homotopy groups of the diffeomorphism groups of discs, spheres and aspherical manifolds, Proc. Sympos. Pure Math., Vol. 32, Amer. Math. Soc., Providence, R.I., 1978, pp. 325-338.

[FH3] . Topological characterization of flat and almost flat Riemannian manifolds $M^{n}(n \neq 3,4)$, Amer. J. Math. 105 (1983), 641-672.

[HS] W.-C. Hsiang and R. Sharpe, Parametrized surgery and isotopy, Pacific J. Math. 67 (1976), 401-459.

[KS] R. C. Kirby and L. C. Siebenmann, Foundational essays on topological manifolds, smoothings and triangulations, Ann. of Math. Studies, No. 88, Princeton Univ. Press, Princeton, N.J., 1977.

[N] A. J. Nicas, Induction theorems for groups of homotopy manifold structures, Mem. Amer. Math. Soc. No. 267 (1982).

[N2] _ On $\mathrm{Wh}_{2}$ of a Bieberbach group, Topoiogy (to appear).

[N3] On $\mathrm{Wh}_{3}$ of a Bieberbach group, Math. Proc. Cambridge Philos. Soc. (to appear).

[O] P. Orlik, Seifert manifolds, Lecture Notes in Math., Vol. 291, Springer-Verlag, Berlin and New York, 1972.

[P] S. P. Plotnick, Vanishing of Whitehead groups for Seifert manifolds with infinite fundamental group, Comment. Math. Helv. 55 (1980), 654-667.

[S] C. W. Stark, Structure sets vanish for certain bundles over Seifert manifolds, Trans. Amer. Math. Soc. (to appear).

[SW] R. G. Swan, K-theory of finite groups and orders, Lecture Notes in Math., Vol. 149, Springer-Verlag, Berlin and New York, 1970.

[W] F. Waldhausen, Algebraic K-theory of generalized free products, Ann. of Math. (2) 108 (1978), $135-256$.

Department of Mathematics, University of Toronto, Toronto, Ontario, Canada M5S 1A1

Department of Mathematics, Brandeis University, Waltham, Massachusetts 02154 